\title{
ON 2-GENERATOR 2-RELATION SOLUBLE GROUPS
}

\author{
by C. M. CAMPBELL and E. F. ROBERTSON
}

(Received 30th October 1978)

The class of non-metacyclic finite soluble groups known to have 2-generator 2-relation presentations is small. Classes of such groups are given in (3), (4), (8) and (9). Some subclasses of the groups discussed in (1) and (2) also provide examples, while a class of finite nilpotent 2-generator 2-relation groups is given by Macdonald in (7).

Any finite deficiency zero group $G$ must have $G / G^{\prime}$ at most 3 -generated (6). The question arises as to whether there is a bound on the rank of other factors of the derived series. For the groups given in (4), (8), and (9) these factors are at most 2 -generated (5). We shall show that examples with $G^{\prime} / G^{\prime \prime}$ of unbounded rank can be found in the class investigated in (2), that is the class

$$
F^{a, b, c}=\left\langle x, y \mid x^{2}=1, x y^{a} x y^{b} x y^{c}=1\right\rangle .
$$

For this purpose it is convenient to define the class

$$
F_{\lambda}^{a, b, c}=\left\langle x, y \mid x^{2}=y^{\lambda(a+b+c)}=x y^{a} x y^{b} x y^{c}\right\rangle,
$$

noting that $F_{0}^{a, b, c}=F^{a, b, c}$, while $F_{2}^{a, b, c}$ is studied in (3).

We first prove some general results concerning $F_{\lambda}^{a, b, c}$. Introduce the notation $n=$ $a+b+c$ and $d=(a-b, b-c, \lambda n)$. The group $F_{\lambda}^{a, b, c}$ has an infinite abelian factor group if $n=0$ or $\lambda=-2$ and, since $F_{\lambda}^{a, b, c}$ is isomorphic to $F_{\lambda}{ }^{a,-b,-c}$, we assume that $n>0$ and $\lambda \neq-2$. Once the case $(a, b, c)=1$ is solved, arguments similar to those in (3) enable the case $(a, b, c) \neq 1$ to be dealt with, so from now on assume $(a, b, c)=1$.

For any non-zero integer $r$ let $C_{r}$ denote the cyclic group of order $|r|$. Putting $G=F_{\lambda}^{a, b, c}$ we have $G / G^{\prime} \cong C_{(\lambda+2) n}$. Define $K_{\lambda}^{a, b, c}$ to be $G^{\prime} / G^{\prime \prime}$.

Lemma 1. $G^{\prime \prime}$ contains $\left\langle y^{(\lambda+2) n}\right\rangle^{G}$, the normal closure of $y^{(\lambda+2) n}$ in $F_{\lambda}^{a, b, c}$.

Proof. In $F_{A}^{a, b, c}$ we have

$$
x^{-1} y^{-a} x=y^{b} x y^{c}
$$

and, since $x^{2}$ is central, two additional relations obtained from (1) by permuting $a, b, c$ cyclically. Now $x y^{n}$ and $x y^{n-a} x y^{a} x^{-1}$ belong to $G^{\prime}$ so $G^{\prime \prime}$ contains

$$
\begin{aligned}
{\left[x y^{n-a} x y^{a} x^{-1}, x y^{n}\right] } & =x y^{-a} x^{-1} y^{-n+a}\left(x^{-1} y^{-a} x\right) y^{a+n} \\
& =x y^{-a}\left(x^{-1} y^{-c} x\right) y^{2 n-b} \\
& =x^{2} y^{2 n} \\
& =y^{(\lambda+2) n}
\end{aligned}
$$


The Reidemeister-Schreier algorithm may be used to find a presentation for the derived group of $F_{\lambda}^{a, b, c} /\left\langle y^{(\lambda+2) n}\right\rangle^{G}$ and this derived group shown to be abelian using a technique similar to that in Section 3 of (2). This proves the first part of the next theorem.

\section{Theorem 2.}

(i) $F_{\lambda}^{a, b, c} /\left\langle y^{(\lambda+2) n}\right\rangle^{G}$ is metabelian.

(ii) $\left\langle y^{(\lambda+2) n}\right\rangle^{G}=G^{\prime \prime}$.

(iii) If $\lambda$ is even, $K_{\lambda}^{a, b, c} \cong K_{0}^{a, b, c}$. $K_{0}^{a, b, c}$.

(iv) If $\lambda$ is odd, $K_{\lambda}^{a, b, c} \cong K_{-1}^{a, b, c}$ and $K_{-1}^{a, b, c}$ is the maximal elementary abelian 2-factor of

Proof. (ii) This is a consequence of (i) and Lemma 1.

(iii) and (iv). By (ii) $K_{\lambda}^{a, b, c}$ is the derived group of $F_{\lambda}^{a, b, c} /\left\langle y^{(\lambda+2) n}\right\rangle^{G}$. The ReidemeisterSchreier method referred to above then shows that $K_{\lambda}^{a, b, c}$ depends on $\lambda$ only to the extent of its parity. For, putting $x_{i}=y^{i-1} x y^{n-i+1}, 1 \leqq i \leqq n$, we obtain the presentation

$$
\left\langle x_{1}, x_{2}, \ldots, x_{n} \mid x_{i+a}^{\varepsilon_{i+a}}=x_{i}^{\varepsilon_{i}} x_{i+a+b}^{\varepsilon_{i+a}}, 1 \leqq i \leqq 2 n\right\rangle
$$

for $K_{\lambda}^{a, b, c}$ when $\lambda$ is even, and

$$
\left\langle x_{1}, x_{2}, \ldots, x_{n} \mid x_{i+a}=x_{i} x_{i+a+b}, x_{i}^{2}=1,1 \leqq i \leqq n\right\rangle
$$

when $\lambda$ is odd. In these two presentations the subscripts on the $x$ 's are reduced modulo $n$ and $\varepsilon_{i}$ is +1 or -1 according to whether $i$ reduced modulo $2 n$ lies in the range 1 to $n$ or $n+1$ to $2 n$ respectively.

To find the rank of $K_{-1}^{a, b, c}$ associate with $F_{-1}^{a, b, c}$ the $n \times n$ matrix $A=\left(a_{i j}\right)$ over the field $\boldsymbol{F}_{2}$ defined by

where

$$
a_{i j}=\delta_{i, j}+\delta_{i+a, j}+\delta_{i+a+b, j}
$$

$$
\delta_{i, j}=\left\{\begin{array}{l}
1 \text { if } i \equiv j(\bmod n), \\
0 \text { otherwise. }
\end{array}\right.
$$

Lemma 3. The elementary abelian 2-group $K_{-1}^{a, b, c}$ has rank $n$ - $t$ where t is the rank of the matrix $A$.

Proof. This is immediate from the presentation for $K_{\lambda}^{a, b, c}, \lambda$ odd, given in the proof of Theorem 2.

We can now show that the rank of $K_{-1}^{a, b, c}$ is unbounded.

Theorem 4. Given any $k \geqq 2$ there exists a positive integer $m \leqq 2^{k}-k-1$ such that $K_{-1}^{1, k-1, m}$ has rank $k$.

Proof. Let $\boldsymbol{F}_{2}^{k}$ be the vector space of $k$-tuples over $\boldsymbol{F}_{2}$ with basis $\left\{e_{i}: 1 \leqq i \leqq k\right\}$ where, as usual, $e_{i}$ denotes the $k$-tuple whose only non-zero entry is in the $i$-th position. Define $\boldsymbol{\theta}: \boldsymbol{F}_{2}^{k} \rightarrow \boldsymbol{F}_{2}^{k}$ by

$$
e_{i} \theta=e_{i+1}, 1 \leqq i \leqq k-1 ; e_{k} \theta=e_{1}+e_{2}
$$


Clearly $\theta$ is an isomorphism so $\theta$ belongs to $G L(k, 2)$ and has order $|\theta|$, say, where $|\theta| \leqq 2^{k}-1$.

Consider $k$ to be fixed. We shall find necessary and sufficient conditions on the positive integer $m$ so that the $(k+m) \times(k+m)$ matrix $A$ associated with $F_{-1}^{1, k-1, m}$ has rank $m$. The rank of $A$ is the same as the rank of the circulant matrix $B$ over $F_{2}$ with first row

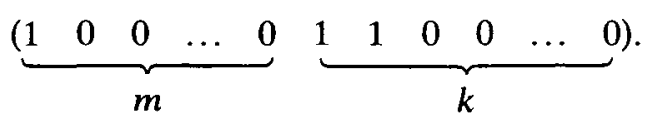

Reduce $B$ to upper triangular form using elementary row operations. It is straightforward to check that after this reduction the final $k$-tuple of entries in row $i$ is $\left(e_{1}+e_{2}\right) \theta^{i-1}$ for $1 \leqq i \leqq m$. The last $k$ rows of the reduced matrix consist entirely of zeros if, and only if, $\left(e_{1}+e_{2}\right) \theta^{m}=e_{1}$. But $e_{1} \theta^{k}=e_{1}+e_{2}$ so the conditions on $m$ are that $m=t-k$ where $t$ is divisible by $|\theta|$. In particular we can choose $m=|\theta|-k$ and the result follows.

\section{Corollary 5. $K_{0}^{a, b, c}$ has unbounded rank.}

Proof. This follows from Theorem 2(iv) and Theorem 4.

To show that the class $F_{\lambda}^{a, b, c}$ contains examples of finite soluble groups with derived groups of unbounded rank it remains to study $G^{\prime \prime}$.

Lemma 6. In $F_{\lambda}^{a, b, c} y^{2 n}$ commutes with $x^{-1} y^{d} x$.

Proof. From (1) $x^{-1} y^{a} x=y^{-c} x^{-1} y^{-b}$ and $x^{-1} y^{b} x=y^{-a} x^{-1} y^{-c}$ so

$$
x^{-1} y^{a-b} x=y^{-c} x^{-1} y^{c-b} x y^{a} \text {. }
$$

By symmetry,

$$
x^{-1} y^{b-c} x=y^{-a} x^{-1} y^{a-c} x y^{b}
$$

and

$$
x^{-1} y^{c-a} x=y^{-b} x^{-1} y^{b-a} x y^{c} \text {. }
$$

Now from (2), (3) and (4)

Hence

$$
x^{-1} y^{a-b} x=y^{-c}\left(y^{-b} x^{-1} y^{c-a} x y^{a}\right) y^{a}=y^{-c-b}\left(y^{-b} x^{-1} y^{b-a} x y^{c}\right) y^{2 a} \text {. }
$$

$$
\begin{aligned}
x^{-1} y^{a-b} x & =y^{-c-2 b}\left(x^{-1} y^{b-a} x\right) y^{c+2 a} \\
& =y^{-c-2 b}\left(y^{-2 a-c} x^{-1} y^{a-b} x y^{c+2 b}\right) y^{c+2 a}
\end{aligned}
$$

and so $\left[y^{2 n}, x^{-1} y^{a-b} x\right]=1$. By symmetry $\left[y^{2 n}, x^{-1} y^{b-c} x\right]=1$ and, since $y^{\lambda n}$ is central, $\left[y^{2 n}, x^{-1} y^{\lambda n} x\right]=1$. Therefore, since $d=(a-b, b-c, \lambda n),\left[y^{2 n}, x^{-1} y^{d} x\right]=1$.

Corollary 7. In $G=F_{\lambda}^{a, b, c}$, if either $d=1$ or $\lambda= \pm 1$ then $G^{\prime \prime}$ is a central cyclic subgroup.

Proof. If $d=1$ then $\left[y^{2 n}, x^{-1} y x\right]=1$ so $\left[y^{2 n}, x^{-1} y^{a} x\right]=1$. Therefore, using (1), $\left[y^{2 n}, x\right]=1$ and $y^{2 n}$ is central. By Theorem 2(ii) $G^{\prime \prime}=\left\langle y^{(\lambda+2) n}\right\rangle^{G}$ so $G^{\prime \prime}$ is a central cyclic subgroup as required. If $\lambda= \pm 1$ then $y^{n}$ is central giving the same result in this case. 
Notice that when $\lambda= \pm 1$ the only possible values of $d$ are 1 and 3 . For, since $(a, b, c)=1$, $d=(a-b, b-c, a+b+c)=(a-b, b-c, 3 a)=(a-b, b-c, 3 a, 3 b, 3 c)=(a-b, b-c, 3)$.

Corollary 8. $F_{\lambda}^{a, b, c}$ is a finite soluble group of derived length $\leqq 3$ if either $d=1$ or $\lambda= \pm 1$.

Proof. In both cases the centre of $F_{\lambda}^{a, b, c}$ has finite index and the commutator quotient of $F_{\lambda}^{a, b, c}$ is finite. Hence the result follows by a well-known theorem due to Schur.

The order of $G^{\prime \prime}$ in the cases $d=1$ and $\lambda= \pm 1$ may be calculated using the Reidemeister-Schreier method. We omit the details of this argument which, now that we have Corollary 7, is similar to that of Theorem 3.3 and Lemma 4.1 of (3). The method shows that $G^{\prime \prime}$ is trivial when $d=1$ and is $C_{2}$ when $d=3$.

The case $d=1$ verifies part of the conjecture given in $\$ 12$ of (2).

The following example shows that the rank of the third factor of the derived series of a finite soluble 2-generator 2-relation group can also exceed 3. Let $H$ be defined by

$$
\left\langle x, y \mid x^{3}=1, y x y^{3} x y^{-3} x=1\right\rangle .
$$

Then $H / H^{\prime} \cong C_{3}$ and the Reidemeister-Schreier method shows that

$$
H^{\prime}=\left\langle x, y, z \mid x y^{3}=z^{3}, y z^{3}=x^{3}, z x^{3}=y^{3}\right\rangle
$$

so that $H^{\prime} / H^{\prime \prime} \cong C_{2} \times C_{14}$. A machine implementation of the Todd-Coxeter coset enumeration algorithm now shows that $H^{\prime \prime}$ is the direct product of seven copies of $C_{2}$.

\section{REFERENCES}

(1) C. M. CAMPBELl, Computational techniques and the structure of groups in a certain class, Proceedings of the 1976 ACM Symposium on Symbolic and Algebraic Computation (1976), 312-321.

(2) C. M. CAMPBEll, H. S. M. COXETER and E. F. ROBERTSON, Some families of finite groups having two generators and two relations, Proc. Roy. Soc. London A 357 (1977), 423-438.

(3) C. M. CAMPBEll and E. F. ROBERTSON, Classes of groups related to $F^{a, b, c}$, Proc. Roy. Soc. Edinburgh 78A (1978), 209-218.

(4) C. M. CAMPBELL and E. F. ROBERTSON, Deficiency zero groups involving Fibonacci and Lucas numbers, Proc. Roy.. Soc. Edinburgh 81A (1978), 273-286.

(5) D. L. JohNSON and E. F. RoBertson, Finite groups of deficiency zero, Proc. Durham Sympos, edited by C. T. C. Wall (LMS Lecture Notes, 36 (1979), 275-289).

(6) D. L. JohNSON, J. W. WAMSLEY and D. WRIGHT, The Fibonacci groups, Proc. London Math. Soc. 29 (1974), 577-592. $602-613$.

(7) I. D. MACDONALD, On a class of finitely presented groups, Canad. J. Math. 14 (1962),

(8) J. W. WAMSLEY, A class of two generator two relation finite groups, J. Australian Math. Soc. 14 (1972), 38-40. 
(9) J. W. WAMSLEY, Some finite groups with zero deficiency, J. Australian Math. Soc. 18 (1974), 73-75.

MATHEMATICAL INSTITUTE

UNIVERSITY OF ST ANDREWS

ST ANDREWS, KY16 9SS 